\title{
Genetic Susceptibility for Ischemic Infarction and Arteriolosclerosis Based on Neuropathologic Evaluations
}

\author{
S.H.-Y. Chou ${ }^{\text {a J.M. Shulman }}{ }^{\text {d, e }}$ B.T. Keenan ${ }^{\text {a }}$ E.A. Secor ${ }^{\text {a }}$ A.S. Buchman ${ }^{\text {b }}$ \\ J. Schneider ${ }^{c}$ D.A. Bennett ${ }^{\text {b }}$ P.L. De Jager ${ }^{a}$ \\ aProgram in Translational NeuroPsychiatric Genomics, Department of Neurology, Brigham and Women's Hospital, \\ Harvard Medical School, Broad Institute at Harvard University and MIT, Boston, Mass., and Departments of \\ ${ }^{b}$ Neurological Sciences and 'Neurology and Neuropathology, Rush University Medical Center, Chicago, III., and \\ ${ }^{\mathrm{d}}$ Departments of Neurology and Molecular and Human Genetics, Baylor College of Medicine, and e Jan and Dan \\ Duncan Neurological Research Institute, Texas Children's Hospital, Houston, Tex., USA
}

\section{Key Words}

Ischemic stroke etiology · Pathology · Genetic risk factors of stroke. Genetics of vascular pathology and stroke.

Diabetes mellitus · Arteriolosclerosis

\begin{abstract}
Background: Recent genetic studies of stroke and related risk factors have identified a growing number of susceptibility loci; however, the relationship of these alleles to ischemic stroke is unknown. The challenge in finding reproducible loci of ischemic stroke susceptibility may be in part related to the etiologic heterogeneity in clinically defined stroke subtypes. In this study, we tested whether known single nucleotide polymorphisms (SNPs) associated with stroke or putative stroke risk factors are associated with neuropathologically defined micro- or macroscopic infarcts and with arteriolosclerosis. Methods: Measures of neuropathology and genotyping were available from 755 deceased participants from the Religious Orders Study and the Rush Memory and Aging Project. All donated brains were examined by a boardcertified neuropathologist using standardized protocol for the presence of microscopic infarct, macroscopic infarct and arteriolosclerosis (lipohyalinosis). In primary analysis, 74 can-
\end{abstract}

didate SNPs previously associated $\left(\mathrm{p}<5 \times 10^{-8}\right)$ with ischemic stroke or known risk factors, including atrial fibrillation $(\mathrm{AF})$, hypertension, diabetes, low-density lipoprotein (LDL) level and carotid artery stenosis, were evaluated for association with neuropathologic endpoints. We performed a secondary exploratory analysis to include 93 additional SNPs associated with putative ischemic stroke risk factors including SNPs associated with high-density lipoprotein (HDL), triglyceride serum levels, myocardial infarction (MI), coronary artery disease and cerebral white matter disease. Regression models relating SNPs to cerebrovascular neuropathology were adjusted for age at death, gender and cohort membership. Results: The strongest associations seen for both macroscopic and microscopic infarcts were risk variants associated with diabetes. The diabetes risk variant $r 57578326$ located near the IRS 1 locus was associated with both macroscopic ( $O R=0.73, p=0.011)$ and microscopic ( $O R=0.71, p=$ 0.009 ) infarct pathology. Another diabetes susceptibility locus (rs 12779790) located between the calcium/calmodulindependent protein kinase ID (CAMK1D) and cell division cycle 123 homolog (CDC123) genes is also associated with both

S.H.-Y. Chou and J.M. Shulman contributed equally to this work.

\section{KARGER}

E-Mail karger@karger.com

www.karger.com/ced
(C) 2013 S. Karger AG, Basel

1015-9770/13/0363-0181\$38.00/0
Philip L. De Jager, MD, PhD

Program in Translational NeuroPsychiatric Genomics

Department of Neurology, Brigham and Women's Hospital

77 Avenue Louis Pasteur, NRB 168C, Boston, MA 02115 (USA)

E-Mail pdejager@ rics.bwh.harvard.edu 
macroscopic ( $O R=1.40, p=0.0292)$ and microscopic infarcts $(\mathrm{OR}=1.43, \mathrm{p}=0.0285)$. The diabetes risk variant $r s 864745$ within JAZF1 was associated with arteriolosclerosis (OR = $0.80, p=0.014)$. We observed suggestive associations with the diabetes risk variant $r s 7961581(\mathrm{p}=0.038$; between TSPAN8 and LGR5) and rs5215 ( $\mathrm{p}=0.043 ; K C N J 11)$, the LDL risk variant $\mathrm{rs} 11206510(\mathrm{p}=0.045 ; P C S K 9)$, as well as the $\mathrm{AF}$ risk locus ZFHX3. The CDKN2A/B locus (rs2383207, 9p21), identified initially as a susceptibility allele for $\mathrm{MI}$ and recently implicated in large vessel stroke, was associated with macroscopic infarct pathology in our autopsy cohort $(\mathrm{OR}=1.26$, $p=0.031)$. Conclusion: Our results suggest replication of the candidate $C D K N 2 A / B$ stroke susceptibility locus with directly measured macroscopic stroke neuropathology, and further implicate several diabetes and other risk variants with secondary, pleiotropic associations to stroke-related pathology in our autopsy cohort. When coupled with larger sample sizes, cerebrovascular neuropathologic phenotypes will likely be powerful tools for the genetic dissection of susceptibility for ischemic stroke.

(c) 2013 S. Karger AG, Basel

\section{Introduction}

Beyond the well-recognized clinical and environmental risk factors, studies point to a substantial heritability for ischemic stroke [1,2]. However, elucidation of genetic risk factors for stroke remains a challenge. Recently, genome-wide association studies (GWAS) have begun to identify several promising susceptibility loci $[3,4]$. In addition, a large number of common susceptibility variants have been identified by GWAS targeting epidemiologically proven stroke risk factors, such as diabetes, hypertension and atrial fibrillation (AF). One potential barrier in the discovery of stroke susceptibility loci is etiologic heterogeneity in stroke subtypes amongst clinically defined cases $[5,6]$. Further, based on neuroimaging and autopsy studies, clinically silent strokes commonly occur in older adults [7], and the presence of a substantial burden of subclinical strokes in clinically defined controls might further confound the discovery of stroke susceptibility loci.

Compared to clinical stroke, the genetics of stroke neuropathology has been largely unexplored. Evaluation of discrete cerebrovascular neuropathologic phenotypes, including micro- or macroscopic infarcts and arteriolosclerosis (lipohyalinosis) might be expected in part to moderate the effects of etiologic confounding in genetic association studies. Further, neuropathologic intermedi- ate phenotypes such as microscopic infarct, macroscopic infarcts and arteriolosclerosis may shed mechanistic insights into the effects of susceptibility variants on more distal clinical diagnoses. Here, we leverage detailed genotype and phenotype data from two large, prospective cohort studies with postmortem organ donation to evaluate the relationship between known susceptibility loci for stroke or ischemic stroke risk factors with cerebrovascular neuropathologic phenotypes.

\section{Methods}

Subjects

Participants were derived from two similar cohort studies: the Religious Orders Study (ROS) and the Rush Memory and Aging Project (MAP). ROS was established in 1994 and enrolls elderly Catholic clergy aged 55 and older and without known dementia from the USA. MAP was established in 1997 and enrolls older men and women without known dementia from retirement centers in the Chicago area. Subjects from both studies are followed longitudinally with annual clinical exams and have signed Anatomic Gift Acts donating their brains after death. We analyzed ROS and MAP subjects jointly, as has been done previously [8-10], for the following reasons: (1) they were designed to be combined, (2) the outcome measures and data collection procedures are identical, and (3) they are managed by a single investigative team. All subjects included in this study are Caucasian, all gave informed consent for study participation and the studies were approved by Rush University Medical Center's Institutional Review Board.

\section{Brain Pathological Evaluation}

All donated brains are removed and examined according to a standardized protocol previously described $[11,12]$. The cerebral and cerebellar hemispheres were cut into $1-\mathrm{cm}$-thick coronal sections. One cerebral and cerebellar hemisphere and the brain stem were fixed in $4 \%$ paraformaldehyde for at least $48 \mathrm{~h}$. All neuropathologic evaluations were systematically performed by a boardcertified neuropathologist at the Rush Alzheimer's Disease Center.

Macroscopic infarcts were defined as any infarct visible to the naked eye, including lacunar infarcts. All visualized and suspected macroscopic infarcts were dissected and confirmed by histology. Primary intraparenchymal hemorrhages were not included in the analysis. Microscopic infarcts were defined as any infarct not visible on gross examination by the naked eye but subsequently visible on hematoxylin- and eosin-stained 6- $\mu \mathrm{m}$ sections under microscopy. Microscopic infarcts on neuropathology likely represent clinical subcortical white matter disease (leukoaraiosis) on neuroimaging [13]. Data for microscopic infarct were derived from examination of the following standardized regions in a single hemisphere: midfrontal, middle temporal, inferior parietal, entorhinal, hippocampal and anterior cingulate cortices, anterior basal ganglia (including caudate, putamen, internal capsule and globus pallidus), anterior thalamus and midbrain. For the purpose of our analysis, microscopic and macroscopic infarcts were coded as present or absent. Because acute and subacute infarcts are often related to perimortem health status, only chronic infarcts were included in 
the analyses. Arteriolosclerosis (or lipohyalinosis) is defined as concentric hyalinization of arteriolar walls with resulting narrowing of the lumen and was evaluated by examining the small penetrating arterioles within the basal ganglia and classified by the board-certified neuropathologist on a semi-quantitative scale ranging from 0 (none) to 7 (completely occluded). For analysis, patients were classified as having none, mild, moderate or severe arteriolosclerosis.

\section{Candidate Single-Nucleotide Polymorphism Selection}

We selected candidate single-nucleotide polymorphisms (SNPs) associated with epidemiologic risk factors for clinical ischemic stroke with a $\mathrm{p}<5 \times 10^{-8}$ from published GWAS studies. Our primary analyses examined 74 SNPs associated with well-established ischemic stroke risk factors, including $\mathrm{AF}$, hypertension, diabetes, low-density lipoprotein (LDL) serum level, carotid artery stenosis and SNPs previously found to be associated with clinical stroke in GWAS. These represent 48 independent SNPs after linkage disequilibrium pruning. We extended this approach by performing exploratory secondary analyses including 93 additional SNPs associated with putative risk factors that are implicated in ischemic stroke, but which have been less definitively established than those considered in primary analyses. The secondary analyses included SNPs associated with high-density lipoprotein (HDL), triglyceride serum levels, myocardial infarction (MI), coronary artery disease $(\mathrm{CAD})$ and cerebral white matter disease. Following linkage disequilibrium pruning, 51 independent SNPs remained. To match our study cohort, we excluded any SNPs from studies on subjects of non-Caucasian ancestry.

\section{Genotyping and Imputation Methods}

Genetic information included genotyped and imputed SNPs. In ROS and MAP, DNA was extracted from whole blood lymphocytes or frozen postmortem brain tissue. Genotype data was generated on self-declared non-Hispanic Caucasians using the Affymetrix Genechip 6.0 platform at the Broad Institute's Genetic Analysis Platform or the Translational Genomics Research Institute. Both sets of data underwent the same quality control analyses in parallel using the PLINK toolkit (http://pngu.mgh.harvard. $\mathrm{edu} / \sim$ purcell/plink/) and quality controlled genotypes were pooled. The quality control process included a principal components analysis using default parameters in EIGENSTRAT [14] to identify and remove population outliers. Imputation in ROS and MAP was performed using MACH software (version 1.0.16a) and HapMap release 22 CEU (build 36). SNPs with an MAF $<1 \%$ and an imputation quality score $<0.3$ were excluded from analyses.

\section{Statistical Methods}

Demographic information was described using means and standard deviations for continuous variables and counts and percentages for categorical variables. Logistic regression was used to assess the association between candidate SNPs and the binary measures (presence or absence) of macroscopic and microscopic infarcts. We used a proportional odds regression model for measure of arteriolosclerosis. All models were adjusted for age at death, sex and cohort. To correct for multiple comparisons, we used the linkage disequilibrium to determine the number of independent SNPs and then performed a Bonferroni correction. At an $\mathrm{r}^{2}$ threshold of 0.1 , we had 48 independent SNPs in our primary analyses ( $p$ value of 0.001 for significance) and 51 independent SNPs in the second-

Genetic Susceptibility for Cerebrovascular

Neuropathology
Table 1. Demographic characteristics of subject cohorts

\begin{tabular}{|c|c|c|c|}
\hline & $\begin{array}{l}\text { ROS } \\
(n=420)\end{array}$ & $\begin{array}{l}\text { MAP } \\
(n=335)\end{array}$ & $\begin{array}{l}\text { All } \\
(\mathrm{n}=755)\end{array}$ \\
\hline Age at death, years & $87.3 \pm 6.9$ & $89.2 \pm 5.4$ & $88.1 \pm 6.3$ \\
\hline Male & $157(37)$ & $119(36)$ & $276(37)$ \\
\hline Macroscopic infarct present & $150(36)$ & $118(35)$ & $268(36)$ \\
\hline Microscopic infarct present & $125(30)$ & $76(23)$ & $201(27)$ \\
\hline \multicolumn{4}{|l|}{ Arteriosclerosis } \\
\hline None & $144(35)$ & $68(20)$ & $212(28)$ \\
\hline Mild & $124(30)$ & $122(37)$ & $246(33)$ \\
\hline Moderate & $94(23)$ & $109(33)$ & $203(27)$ \\
\hline Severe & $50(12)$ & $35(10)$ & $85(11)$ \\
\hline \multicolumn{4}{|l|}{ Disease history } \\
\hline Diabetes & $84(20)$ & $65(19)$ & $149(20)$ \\
\hline Stroke & $105(25)$ & $89(27)$ & $194(26)$ \\
\hline Hypertension & $224(54)$ & $214(64)$ & $438(58)$ \\
\hline
\end{tabular}

Values in parentheses are percentages.

ary analyses ( $\mathrm{p}$ value of 0.001 for significance). Due to the exploratory nature of these analyses, we considered anything with a $\mathrm{p}<$ 0.05 to be a suggestive association. Statistical analyses were performed using SAS 9.1 (SAS Institute, Cary, N.C., USA), the PLINK toolkit (http://pngu.mgh.harvard.edu/ purcell/plink/) and $\mathrm{R}$ (version 2.13; www.r-project.org).

\section{Results}

Demographic details of the study cohorts are summarized in table 1 . The mean age at death was 88 years and $63 \%$ were female. At autopsy, $36 \%$ had macroscopic infarcts, $27 \%$ had microscopic infarcts and $38 \%$ had moderate or severe arteriolosclerosis. Overall, $47 \%$ of the study cohort had evidence of either micro- or macroscopic infarct pathology at autopsy.

Overall, 211 out of 816 (26\%) of our cohort had a clinical history of stroke (table 2). Subjects who had a history of clinical strokes were more likely to have macroscopic and microscopic infarcts, as well as higher severity of arteriolosclerosis. Clinically, subjects with history of clinical stroke were more likely to have a history of hypertension.

\section{Macroscopic and Microscopic Infarcts}

Table 3 reports the results of our primary analyses meeting our suggestive significance criteria ( $\mathrm{p}<0.05$; full results are displayed in the online suppl. table $\mathrm{S} 1$; see www.karger.com/doi/10.1159/000352054 for all online suppl. material). The differential association of macro- 
scopic and microscopic infarctions with SNPs selected for primary analysis stratified by cortical versus subcortical locations are summarized in online suppl. table S6. In secondary analyses, we further evaluated an additional 51 independent SNPs with several additional traits putatively linked to stroke pathogenesis, including LDL, triglyceride levels, history of MI, CAD and cerebral white matter disease (table 4; online suppl. table S2). These analyses were considered exploratory as the selected traits lack the same strength of epidemiological evidence for relation with stroke as those in the primary analyses. However, all SNPs were similarly selected by stringent genome-wide significant associations in the published genetics literature. As detailed below, our analyses identified several suggestive associations with stroke neuropathologic traits; however, no SNP remained significant in either our primary or secondary analyses following adjustment for multiple tests.

Based on published GWAS, only three susceptibility loci, NINJ2, PITX2 and HDAC9, achieved genome-wide significance criteria for clinically defined stroke $[4,15]$. Published SNPs at these loci were not associated (online suppl. table S2) with any of the neuropathologic traits considered in our analyses. Two additional loci, ZFHX3 and $C D K N 2 A / B$, are strong candidate stroke susceptibility loci, based on initial discovery of significant associations with $\mathrm{AF}$ and $\mathrm{MI}$, respectively, and subsequent highly suggestive evidence of association $\left(\mathrm{p}<10^{-4}\right)$ with clinical stroke $[16,17]$. We found that the ZFXH3 SNP, rs7193343, had evidence of association with arteriolosclerosis, whereas $r s 2383207$ at the $C D K N 2 A / B$ locus was associated with macroscopic infarct pathology (table 3,4).

By contrast with the few established susceptibility loci for clinical strokes, numerous genetic variants have been validated for association with proven stroke risk factors, and several also demonstrate evidence of association with the stroke neuropathologic traits that we have studied. The strongest association seen for both macroscopic and microscopic stroke pathology was the chromosome 2 diabetes risk variant $r s 7578326$ (table 3) [18]. This SNP is in an intergenic region between the neuronal tyrosine-phosphorylated phosphoinositide-3-kinase adaptor 2 (NYAP2) and the insulin receptor substrate 1 (IRS1) genes. IRS1 encodes a substrate and key signal transduction component downstream of the insulin receptor. Another diabetes susceptibility locus, $r s 12779790$ [19], was associated with both micro- and macroscopic infarct pathology (table 3 ). This chromosome 10 variant is between the calcium/ calmodulin-dependent protein kinase ID (CAMK1D) and cell division cycle 123 homolog (CDC123) genes.
Table 2. Neuropathologic characteristics and clinical histories of the cohort stratified by clinical history of stroke

\begin{tabular}{lccc}
\hline & $\begin{array}{c}\text { No history } \\
\text { of stroke } \\
(\mathrm{n}=211)\end{array}$ & $\begin{array}{l}\text { History } \\
\text { of stroke } \\
(\mathrm{n}=605)\end{array}$ & p value \\
& $159(29.3)$ & $105(54.7)$ & $<0.001$ \\
Macroscopic infarcts & $44(8.2)$ & $45(23.7)$ & $<0.001$ \\
Cortical & $130(24.1)$ & $79(41.6)$ & $<0.001$ \\
Subcortical & $134(24.7)$ & $67(34.9)$ & 0.007 \\
Microscopic infarcts & $71(13.2)$ & $30(15.8)$ & 0.37 \\
Cortical & $75(13.9)$ & $42(22.1)$ & 0.008 \\
Subcortical & $70(13.1)$ & $19(10.2)$ & \\
Cerebral vascular disease atherosclerosis rating (CVDA) & 0.09 \\
0 & $231(43.3)$ & $69(36.9)$ & \\
1 & $184(34.5)$ & $73(39.0)$ & \\
2 & $48(9.0)$ & $26(13.9)$ & \\
3 & $161(30.0)$ & $45(23.9)$ & \\
Arteriolosclerosis (lipohyalinosis) & $178(33.2)$ & $59(31.4)$ & \\
None & $146(27.2)$ & $51(27.1)$ & \\
Mild & $52(9.7)$ & $33(17.6)$ & \\
Moderate & $116(19.2)$ & $52(24.6)$ & 0.09 \\
Severe & $249(42.1)$ & $106(51.7)$ & 0.02 \\
History of diabetes & & & \\
History of hypertension & &
\end{tabular}

Values in parentheses are percentages.

In addition to the SNPs with consistent associations across phenotypes, we discovered more selective associations between macroscopic stroke pathology and several susceptibility loci (table 3,4), including another diabetes risk variant $r s 1470579$ (IGF2BP2) [18], several LDL SNPs, rs3846663 (HMGCR), rs2075650 (APOE/TOMM40) and rs 12740374 (CELSR2) [20, 21], and the hypertension susceptibility allele $r s 2681472(\mathrm{p}=0.030, A T P 2 B 1)$ [22]. Only one SNP, rs4457053 (LOC728723), was selectively associated with microscopic stroke pathology, and this variant was initially reported in association with diabetes [18].

\section{Arteriolosclerosis}

The strongest association with arteriolosclerosis pathology in our primary analysis was the chromosome 7 diabetes risk variant $r s 864745(\mathrm{p}=0.014)$ within the JAZF zinc finger 1 (JAZF1) gene (table 3 ). We also observed suggestive associations with the diabetes risk variants rs7961581 ( $\mathrm{p}=0.038$; between TSPAN8 and LGR5) and rs5215 ( $\mathrm{p}=0.043 ; K C N J 11)$, the LDL risk variant rs 11206510 ( $\mathrm{p}=0.045 ;$ PCSK9), as well as the AF risk locus $Z F H X 3$ (table 3 ). 
Table 3. Suggestive independent associations for candidate SNPs in primary analyses

\begin{tabular}{|c|c|c|c|c|c|c|c|c|c|}
\hline \multirow[t]{2}{*}{ CHR } & \multirow[t]{2}{*}{ SNP } & \multirow[t]{2}{*}{ Allele } & \multirow[t]{2}{*}{ GWAS } & \multicolumn{2}{|c|}{ Macroscopic infarcts } & \multicolumn{2}{|c|}{ Microscopic infarcts } & \multicolumn{2}{|l|}{ Arteriolosclerosis } \\
\hline & & & & OR $(95 \% \mathrm{CI})$ & $\mathrm{p}$ & OR $(95 \% \mathrm{CI})$ & $\mathrm{p}$ & OR (95\% CI) & $\mathrm{p}$ \\
\hline 2 & rs7578326 & $\mathrm{A}$ & DMII & $0.73(0.58,0.93)$ & 0.0110 & $0.71(0.55,0.92)$ & 0.0088 & $0.90(0.73,1.11)$ & 0.3375 \\
\hline 5 & rs3846663 & $\mathrm{T}$ & LDL & $1.30(1.04,1.63)$ & 0.0212 & $1.11(0.87,1.41)$ & 0.4083 & $1.08(0.89,1.31)$ & 0.4230 \\
\hline 3 & rs1470579 & $\mathrm{C}$ & DMII & $1.32(1.04,1.68)$ & 0.0222 & $1.21(0.94,1.57)$ & 0.1365 & $1.04(0.85,1.28)$ & 0.6794 \\
\hline 19 & rs2075650 & G & LDL & $1.64(1.07,2.52)$ & 0.0241 & $1.46(0.93,2.30)$ & 0.0976 & $1.14(0.79,1.66)$ & 0.4843 \\
\hline 10 & rs12779790 & G & DMII & $1.40(1.03,1.89)$ & 0.0292 & $1.43(1.04,1.96)$ & 0.0285 & $1.03(0.79,1.34)$ & 0.8450 \\
\hline 12 & rs2681472 & $\mathrm{A}$ & HYP & $1.38(1.03,1.86)$ & 0.0304 & $0.98(0.72,1.32)$ & 0.8787 & $0.96(0.75,1.23)$ & 0.7579 \\
\hline 1 & rs 12740374 & G & LDL & $1.33(1.01,1.75)$ & 0.0422 & $1.15(0.86,1.54)$ & 0.3400 & $1.11(0.88,1.39)$ & 0.3781 \\
\hline 5 & rs4457053 & G & DMII & $0.85(0.63,1.15)$ & 0.2876 & $0.71(0.51,0.99)$ & 0.0441 & $0.86(0.67,1.11)$ & 0.2520 \\
\hline 7 & rs864745 & $\mathrm{T}$ & DMII & $0.91(0.73,1.12)$ & 0.3620 & $1.00(0.80,1.26)$ & 0.9939 & $0.80(0.66,0.96)$ & 0.0142 \\
\hline 12 & rs7961581 & $\mathrm{C}$ & DMII & $1.03(0.81,1.32)$ & 0.7941 & $1.10(0.85,1.43)$ & 0.4732 & $1.26(1.01,1.56)$ & 0.0382 \\
\hline 16 & rs7193343 & $\mathrm{T}$ & $\mathrm{AF}$ & $1.10(0.80,1.51)$ & 0.5526 & $1.08(0.77,1.52)$ & 0.6524 & $0.75(0.57,0.99)$ & 0.0392 \\
\hline 11 & rs5215 & $\mathrm{C}$ & DMII & $0.98(0.79,1.22)$ & 0.8758 & $1.03(0.82,1.30)$ & 0.7896 & $0.82(0.68,0.99)$ & 0.0428 \\
\hline 1 & rs11206510 & $\mathrm{T}$ & LDL & $0.91(0.69,1.21)$ & 0.5217 & $1.10(0.81,1.49)$ & 0.5465 & $1.28(1.01,1.63)$ & 0.0451 \\
\hline
\end{tabular}

Values in bold meet our suggestive significance criteria $(\mathrm{p}<0.05)$. DMII = Type II diabetes mellitus; HYP = hypertension; CHR = chromosome.

Table 4. Suggestive independent associations for candidate SNPs in exploratory analyses

\begin{tabular}{|c|c|c|c|c|c|c|c|c|c|}
\hline \multirow[t]{2}{*}{ CHR } & \multirow[t]{2}{*}{ SNP } & \multirow[t]{2}{*}{ Allele } & \multirow[t]{2}{*}{ GWAS } & \multicolumn{2}{|c|}{ Macroscopic infarcts } & \multicolumn{2}{|c|}{ Microscopic infarcts } & \multicolumn{2}{|l|}{ Arteriolosclerosis } \\
\hline & & & & OR (95\% CI) & $\mathrm{p}$ & OR (95\% CI) & $\mathrm{p}$ & OR (95\% CI) & $\mathrm{p}$ \\
\hline 18 & rs4939883 & $\mathrm{T}$ & HDL & $0.68(0.51,0.92)$ & 0.0116 & $0.89(0.65,1.21)$ & 0.4569 & $0.93(0.73,1.19)$ & 0.5873 \\
\hline 16 & rs1864163 & A & HDL & $1.59(1.08,2.34)$ & 0.0179 & $1.25(0.83,1.88)$ & 0.2818 & $1.16(0.83,1.63)$ & 0.3710 \\
\hline 17 & rs12936587 & G & CAD & $1.29(1.03,1.61)$ & 0.0262 & $1.16(0.91,1.47)$ & 0.2295 & $1.04(0.86,1.26)$ & 0.6824 \\
\hline 8 & rs2954029 & $\mathrm{A}$ & TG & $0.98(0.79,1.21)$ & 0.8196 & $0.74(0.58,0.92)$ & 0.0084 & $0.97(0.81,1.17)$ & 0.7546 \\
\hline 15 & rs3825807 & $\mathrm{A}$ & CAD & $0.99(0.79,1.24)$ & 0.9025 & $0.78(0.61,0.99)$ & 0.0437 & $0.86(0.71,1.05)$ & 0.1330 \\
\hline 11 & rs7395662 & G & HDL & $0.98(0.79,1.22)$ & 0.8611 & $1.13(0.89,1.43)$ & 0.3097 & $0.81(0.67,0.97)$ & 0.0244 \\
\hline
\end{tabular}

Values in bold meet our suggestive significance criteria $(\mathrm{p}<0.05)$. CHR $=$ Chromosome; TG $=$ triglyceride level.

\section{Exploratory Analyses}

As detailed in table 4, our exploratory analyses identified a number of additional suggestive associations with stroke neuropathologic traits. Several susceptibility loci were associated with macroscopic infarct pathology, including SNPs previously associated with HDL SNPs, rs1883025 ( $\mathrm{p}=0.009 ; A B C A 1), r s 4939883(\mathrm{p}=0.012$; LIPG) and $r s 1864163(\mathrm{p}=0.018$; CETP), the CAD risk variants, $r s 12936584(\mathrm{p}=0.026 ; K R T A P 2-1$ and KRTAP2-2) and rs46522 ( $\mathrm{p}=0.049 ; U B E 2 Z)$, as well as the MI risk variant, $r s 2383207$, discussed above. The mi- croscopic stroke pathologic trait was associated with the triglyceride level susceptibility SNP $r s 2954029(\mathrm{p}=0.008$; TRIB1) and a CAD variant, $r s 3825807(\mathrm{p}=0.044 ; A D$ AMTS7). Finally, the HDL risk variant, rs7395662, was associated with arteriolosclerosis $(\mathrm{p}=0.024 ;$ OR4A46P).

A number of risk loci from diabetes GWAS were associated with infarct-related pathology in our analyses. It is possible that these genetic associations are 'mediated' by the effect of polymorphisms on diabetes or rather via an independent causal pathway. In the ROS/MAP cohort, $20 \%$ of subjects had a known medical history of diabetes 
prior to death, and a history of diabetes was associated with increased odds of macroscopic infarct on neuropathology [OR = $1.54(1.07-2.21)$; online suppl. table S3]. In order to distinguish between these possibilities, we performed a statistical mediation analysis (online suppl. table S4a, b). We included a covariate for diabetes diagnosis in our regression models relating SNPs (from diabetes GWAS) to the stroke neuropathologic traits. This adjustment did not alter the strength, direction or significance of these associations. We obtained similar results when adjusting analyses of hypertension risk alleles for this diagnosis.

We also explored the effect of history of clinical stroke on the association of candidate SNPs to neuropathologic phenotypes by repeating our primary analysis stratified by history of clinical stroke (online suppl. table S5). Many candidate SNPs identified with potential association to neuropathologic phenotypes in the primary analysis were also seen in this stratified analysis.

\section{Discussion}

We have tested validated susceptibility loci for stroke or ischemic stroke risk factors for associations with cerebrovascular neuropathologic phenotypes in a large, prospective autopsy cohort. Our findings identify several suggestive associations between these candidate variants and either micro- or macroscopic infarcts or arteriolosclerosis. Although none of these associations remained significant following multiple test correction, they are excellent targets for further evaluation in additional cohorts with similar pathologic characterization. Our results further suggest that cerebrovascular neuropathologic traits may enhance genetic analyses of ischemic stroke susceptibility based on clinical diagnoses and also reinforce the emerging pleiotropic nature of the genetic architecture of complex human traits.

Compared to the genetic analyses of pertinent risk factors of ischemic stroke, GWAS of stroke susceptibility have identified relatively few loci to date, despite strong evidence from epidemiology of substantial heritability for this trait $[1,2,4,23]$. One major contributor is heterogeneity within the ischemic stroke phenotype, as distinct stroke mechanisms may have largely nonoverlapping genetic risk profiles. Indeed, large epidemiologic studies have suggested that ischemic stroke subtypes are associated with different recurrence risk, outcome, treatment response and risk factor profiles [24-26], likely mirroring a diversity of underlying pathophysiology. Consistent with this, in one recent stroke GWAS [15], a pooled analysis failed to identify significantly associated loci, whereas differentiation of large vessel and cardioembolic mechanisms uncovered a number of susceptibility variants based on stringent significance criteria. Besides heterogeneity amongst cases, another potential confounder of a clinical case/control GWAS analysis is the common occurrence of subclinical stroke pathology in older adults, as such subjects would be inadvertently misclassified within the control group.

By contrast with clinically defined stroke, our study was based on directly measured macroscopic or microscopic infarct pathology and burden of arteriolosclerosis. This strategy offers potential advantages both with regard to establishing a more homogeneous case population and accounting for subclinical disease in controls. Our comprehensive evaluation of a large number of susceptibility variants, including both clinical stroke as well as proven or putative stroke risk factors, allowed us to evaluate whether distinct risk factor profiles may exist for the neuropathologic subtypes of cerebral infarcts.

We did not find evidence to support associations between established stroke susceptibility variants (HDAC9, PITX2 or NINJ2) from GWAS and infarct-related pathology in ROS/MAP. This is consistent with other reports [27-29]. By contrast, the broader list of evaluated susceptibility alleles, based on GWAS for proven or putative risk factors for ischemic stroke did identify several suggestive associations. Among these findings, two loci, $Z F H X 3$ and $C D K N 2 A / B$, stand out based on being initially discovered for significant associations with $\mathrm{MI}$ and $\mathrm{AF}$, respectively, and later associated with stroke susceptibility. With improved statistical power and larger case/control cohorts in the future, both loci may be established as risk factors for ischemic stroke. In our analyses, we found that the ZFHX3 locus is associated with burden of arteriolosclerosis but not with macro- or microscopic infarcts, while SNPs associated with micro- and macroscopic infarcts are not associated with burden of arteriolosclerosis. This differential association may be due to our limited sample size or, alternatively, may suggest the possibility of divergent genetic susceptibilities and distinct pathophysiologic processes leading to micro- and macroscopic infarcts and to arteriolosclerosis.

Our study has a number of limitations. Given our current sample size and the modest effects of the candidate variants, we had limited power to observe a significant association. Based on the minor allele frequency and effect size observed for our top result, $r s 7578326$, we had only $30 \%$ power to detect a significant effect at our conservative 
Bonferroni-corrected threshold of $\mathrm{p}<0.001$. As a result, our negative results should be interpreted with caution. The ROS and MAP studies are ongoing and continue to accrue autopsy cases, which will allow better powered studies in the future. Collection of similar neuropathologic traits in other community-based, prospective autopsy cohorts may also allow more powerful joint meta-analytic approaches. Another limitation in our study was that the assessment of microscopic infarcts did not include examination of the lower brainstem and cerebellum. Further, because acute infarcts can result from terminal and perimortem health conditions, we limited the analyses to chronic infarcts. There may be differing genetic risk factors for acute infarcts related to mortality. In this patient cohort, we do not have information on cervical and cerebral large artery stenosis or atherosclerosis, and this limits our ability to examine subgroups based on potential large artery stroke etiology. Though we had included SNPs associated with cervical artery atherosclerosis in our initial selection for candidate SNPs, these SNPs were not included in the final analysis as they did not meet the selection criteria for genome-wide significance at $10^{-8}$ level. Due to the nature of this autopsy study, many of the ischemic infarcts included were clinically silent events, and are not easily classified using standard stroke classifications such as TOAST or Oxford criteria. This limits our ability to correlate our findings with clinical stroke subtypes. Finally, we note that many clinical variables of interest constituting important epidemiologic factors affecting stroke risk were not available in this cohort.

Despite its limitations, our study represents, to our knowledge, the first genetic analysis examining variant associations with directly measured stroke-related neuropathology. Though the overall sample size is modest, we observe many suggestive associations that merit evaluation in larger cohorts, including the potential validation of the CDKN2A/B stroke susceptibility locus with macroscopic infarct pathology. Given the heterogeneity found in clinical cohorts of the study of stroke, we suggest that neuropathologic endophenotypes such as those used here can complement and enhance studies of genetic susceptibility for stroke based on clinical case/control samples.

\section{Acknowledgements}

The authors are grateful to the participants of the Religious Orders Study and the Memory and Aging Project. This work is supported by grants from the National Institutes of Health (R01 AG30146, P30 AG10161, R01 AG17917, R01 AG15819, K08 AG034290, K23NS073806, and R01 AG11101, P30 AG19610, R01 AG023193, R01 NS059873, P50 AG16574, U01 AG016976, U24 NS051872, P50 AG23173, K01 AG024079), the Illinois Department of Public Health, and the Burroughs Wellcome Fund.

\section{References}

1 Lanktree MB, Dichgans M, Hegele RA: Advances in genomic analysis of stroke: what have we learned and where are we headed? Stroke 2010;41:825-832.

2 Dichgans M: Genetics of ischaemic stroke. Lancet Neurol 2007;6:149-161.

$>3$ Bellenguez C, Bevan S, Gschwendtner A, et al: Genome-wide association study identifies a variant in hdac9 associated with large vessel ischemic stroke. Nat Genet 2012;44:328-333.

4 Ikram MA, Seshadri S, Bis JC, et al: Genomewide association studies of stroke. N Engl J Med 2009;360:1718-1728.

5 Pruissen DM, Kappelle LJ, Rosendaal FR, Algra A: Genetic association studies in ischaemic stroke: replication failure and prospects. Cerebrovasc Dis 2009;27:290-294.

-6 Flossmann E, Schulz UG, Rothwell PM: Potential confounding by intermediate phenotypes in studies of the genetics of ischaemic stroke. Cerebrovasc Dis 2005;19:1-10.

$>7$ Vermeer SE, Longstreth WT Jr, Koudstaal PJ: Silent brain infarcts: a systematic review. Lancet Neurol 2007;6:611-619.
Bennett DA, De Jager PL, Leurgans SE, Schneider JA: Neuropathologic intermediate phenotypes enhance association to Alzheimer susceptibility alleles. Neurology 2009;72: 1495-1503.

$\checkmark 9$ Bennett DA, Schneider JA, Arvanitakis Z, Kelly JF, Aggarwal NT, Shah RC, Wilson RS: Neuropathology of older persons without cognitive impairment from two communitybased studies. Neurology 2006;66:1837-1844.

$>10$ Chibnik L, Shulman J, Leurgans S, Schneider J, Wilson R, Tran D, Aubin C, Buchman A, Heward C, Myers A, Hardy J, Huentelman M, Corneveaux J, Reiman E, Evans D, Bennett D, De Jager P: CR1 is associated with amyloid plaque burden and age-related cognitive decline. Ann Neurol 2011;69:560-569.

$\checkmark 11$ Schneider JA, Wilson RS, Cochran EJ, Bienias JL, Arnold SE, Evans DA, Bennett DA: Relation of cerebral infarctions to dementia and cognitive function in older persons. Neurology 2003;60:1082-1088.
12 Arvanitakis Z, Leurgans SE, Barnes LL, Bennett DA, Schneider JA: Microinfarct pathology, dementia, and cognitive systems. Stroke 2011;42:722-727.

-13 Longstreth WT Jr, Dulberg C, Manolio TA, Lewis MR, Beauchamp NJ Jr, O'Leary D, Carr J, Furberg CD: Incidence, manifestations, and predictors of brain infarcts defined by serial cranial magnetic resonance imaging in the elderly: the cardiovascular health study. Stroke 2002;33:2376-2382.

14 Price AL, Patterson NJ, Plenge RM, Weinblatt ME, Shadick NA, Reich D: Principal components analysis corrects for stratification in genome-wide association studies. Nat Genet 2006;38:904-909.

15 Bellenguez C, Bevan S, Gschwendtner A, et al: Genome-wide association study identifies a variant in HDAC9 associated with large vessel ischemic stroke. Nat Genet 2012;44:328-333.

16 Gudbjartsson DF, Holm H, Gretarsdottir S, et al: A sequence variant in ZFHX3 on 16q22 associates with atrial fibrillation and ischemic stroke. Nat Genet 2009;41:876-878. 
17 Liu Y, Sanoff HK, Cho H, Burd CE, Torrice C, Mohlke KL, Ibrahim JG, Thomas NE, Sharpless NE: INK4/ARF transcript expression is associated with chromosome 9p21 variants linked to atherosclerosis. PLoS One 2009;4:e5027.

-18 Voight BF, Scott LJ, Steinthorsdottir V, et al: Twelve type 2 diabetes susceptibility loci identified through large-scale association analysis. Nat Genet 2010;42:579-589.

19 Zeggini E, Scott LJ, Saxena R, et al: Meta-analysis of genome-wide association data and large-scale replication identifies additional susceptibility loci for type 2 diabetes. Nat Genet 2008;40:638-645.

20 Kathiresan S, Willer CJ, Peloso GM, et al: Common variants at 30 loci contribute to polygenic dyslipidemia. Nat Genet 2009;41: 56-65.
21 Middelberg RP, Ferreira MA, Henders AK, Heath AC, Madden PA, Montgomery GW, Martin NG, Whitfield JB: Genetic variants in LPL, OASL and TOMM40/APOE-C1-C2-C4 genes are associated with multiple cardiovascular-related traits. BMC Med Genet 2011;12: 123.

22 Levy D, Ehret GB, Rice K, et al: Genome-wide association study of blood pressure and hypertension. Nat Genet 2009;41:677-687.

23 Bellenguez C, Bevan S, Gschwendtner A, et al: Genome-wide association study identifies a variant in HDAC9 associated with large vessel ischemic stroke. Nat Genet 2012;44:328-333.

24 Jackson C, Sudlow C: Are lacunar strokes really different? A systematic review of differences in risk factor profiles between lacunar and nonlacunar infarcts. Stroke 2005;36:891901.

25 Ohira T, Shahar E, Chambless LE, Rosamond WD, Mosley TH Jr, Folsom AR: Risk factors for ischemic stroke subtypes: the atherosclerosis risk in communities study. Stroke 2006; 37:2493-2498.
6 Jackson CA, Hutchison A, Dennis MS, Wardlaw JM, Lindgren A, Norrving B, Anderson CS, Hankey GJ, Jamrozik K, Appelros P, Sudlow CL: Differing risk factor profiles of ischemic stroke subtypes: evidence for a distinct lacunar arteriopathy? Stroke 2010;41:624629.

27 Nalls MA, Biffi A, Matarin M, et al: Failure to validate association between $12 \mathrm{p} 13$ variants and ischemic stroke. N Engl J Med 2010;362: 1547-1550.

28 Olsson S, Melander O, Jood K, Smith JG, Lovkvist H, Sjogren M, Engstrom G, Norrving $B$, Lindgren A, Jern C: Genetic variant on chromosome 12p13 does not show association to ischemic stroke in 3 Swedish case-control studies. Stroke 2011;42:214-216.

29 Ding H, Tu X, Xu Y, Xu C, Wang X, Cui G, Bao X, Hui R, Wang QK, Wang DW: No evidence for association of $12 \mathrm{p} 13$ SNPs rs11833579 and rs12425791 within NINJ2 gene with ischemic stroke in Chinese Han population. Atherosclerosis 2011;216:381382 